\title{
Properties of superconductor/ferromagnet structures with spin-dependent scattering
}

\author{
M. Fauré, ${ }^{1}$ A. I. Buzdin, ${ }^{1,2}$ A. A. Golubov,${ }^{3}$ and M. Yu. Kupriyanov ${ }^{4}$ \\ ${ }^{1}$ Centre de Physique Moléculaire Optique et Hertzienne, Université Bordeaux 1-UMR 5798, CNRS, F-33405 Talence Cedex, France \\ ${ }^{2}$ Institut Universitaire de France, Paris, France \\ ${ }^{3}$ Faculty of Science and Technology, University of Twente, 7500 AE Enschede, The Netherlands \\ ${ }^{4}$ Nuclear Physics Institute, Moscow State University, Moscow, 119992 Russia
}

(Received 29 July 2005; revised manuscript received 15 December 2005; published 8 February 2006)

\begin{abstract}
We investigate superconductor/ferromagnet $(\mathrm{S} / \mathrm{F})$ hybrid structures in the dirty limit, described by the Usadel equations. More precisely, the oscillations of the critical temperature and critical current with the thickness of the ferromagnetic layers are studied. We show that spin-flip and spin-orbit scattering lead to the decrease of the decay length and the increase of the oscillation period. The critical current decay is more sensitive to these pair-breaking mechanisms than that of the critical temperature. These two scattering mechanisms should be taken into account to get a better agreement between experimental results and theoretical descriptions. We also study the influence of the interface transparency on the properties of S/F structures.
\end{abstract}

DOI: 10.1103/PhysRevB.73.064505

\section{INTRODUCTION}

It has been known for quite a long time that superconductivity (S) and ferromagnetism (F) are two antagonistic orderings and that they can hardly coexist in the same compound. Although they tend to avoid each other, their mutual interaction may be studied when they are spatially separated, as is realized in superconductor/ferromagnet $(\mathrm{S} / \mathrm{F})$ hybrid structures (as a review, see Refs. 1-3). Indeed, in such systems, superconductivity and ferromagnetism can influence each other through the so-called proximity effect. The main peculiarity of the proximity effect in S/F structures is the damped oscillatory behavior of the superconducting correlations in the F layers (while they monotonically decay in normal layers of superconductor/normal metal structures). In the dirty limit and for large exchange field, the characteristic lengths of the decay and oscillations are the same. They are given by $\xi_{f}=\sqrt{D_{f} / h}$, where $D_{f}$ is the diffusion coefficient in the ferromagnet and $h$ is the exchange field acting on the electron spins. This unusual proximity effect leads to several striking phenomena, such as the nonmonotonic dependence of the critical temperature and current of $\mathrm{S} / \mathrm{F}$ multilayers on the $\mathrm{F}$ layer thickness and the realization of the so-called $\pi$ junction in $\mathrm{S} / \mathrm{F} / \mathrm{S}$ trilayers. ${ }^{1-3}$

Although the existing theory provides a rather good qualitative description of the observed effects, there is still no complete quantitative agreement with experiments. This indicates that besides the exchange field, some additional pairbreaking mechanisms are present in the F layers. Indeed, the spin-flip process is inherent to the ferromagnetic layers (because of magnetic impurities, spin waves, or nonstoichiometric lattices) and may have dramatic consequences on superconductivity (in contrast to nonmagnetic impurities which have very little impact). Such a pair-breaking mechanism also arises in the usually used weak ferromagnetic alloys, because they are close to ferromagnetism disappearance and thus quite favorable to large magnetic disorder. This can be inferred, for instance, from the very strong decrease of the critical current of $S / F / S$ junctions as a function of the thickness of the ferromagnetic layer in experimental studies. ${ }^{4-6}$ In such experiments, the ferromagnetic alloys used were
PACS number(s): 74.50. + r, 75.30.Et
$\mathrm{Cu}_{x} \mathrm{Ni}_{1-x}$ with $x \sim 0.5$, the limiting range of concentration for ferromagnetic properties. In addition, the pair destruction due to spin-orbit interaction must be taken into account as well.

Though the spin-flip and spin-orbit interactions in the ferromagnetic material were introduced in several recent theoretical studies on the proximity effect in S/F hybrids, only a few simple limiting cases were considered (we will give appropriate references below). Thus the problem of a quantitative description of these effects in S/F multilayered systems is still unsolved. In this paper we present the results of a detailed theoretical study of the influence of spin-flip and spin-orbit scattering mechanisms on the critical temperature $\left(T_{c}^{*}\right)$ and critical current $\left(I_{c}\right)$ of S/F multilayered systems. We obtain analytical and numerical solutions of the problem which provide the basis not only for qualitative understanding of experimental results but also to fit the data quantitatively. In particular, we show that the spin-orbit and spin-flip scattering mechanisms influence differently the properties of $\mathrm{S} / \mathrm{F}$ structures: the spin-orbit mechanism can destroy the $T_{c}^{*}$ and $I_{c}$ oscillations while spin-flip scattering can only modify them. We also report on a striking nonintuitive behavior that the critical temperature and current can acquire with variation of the S/F interface transparency.

\section{LINEARIZED USADEL EQUATIONS}

A very convenient set of equations for an inhomogeneous superconductor was elaborated by Eilenberger. ${ }^{7}$ However, the Eilenberger equations can be replaced by the much simpler Usadel equations ${ }^{8}$ when the electron scattering free path in $S / F$ systems is shorter than the superconducting length, which is often the case. These equations are nonlinear but can be simplified when the temperature is close to the critical temperature $T_{c}$ or at any temperature in the F layer when the transparency is low. We consider a $\mathrm{S} / \mathrm{F}$ multilayered system where all physical quantities depend only on the coordinate $x$ perpendicular to the layers. The natural choice of the spinquantization axis is along the direction of the exchange field. In the general case, magnetic and spin-orbit scatterings mix 
up the up- and down-spin states. Therefore, two anomalous Green functions, namely, $F_{+} \sim\left\langle\psi_{\uparrow} \psi_{\downarrow}\right\rangle$ and $F_{-} \sim\left\langle\psi_{\downarrow} \psi_{\uparrow}\right\rangle$, are needed to describe this situation. The linearized Usadel equations may be written for $\omega>0$ as ${ }^{1,9}$

$$
\left(\omega-\frac{D_{s}}{2} \frac{\partial^{2}}{\partial x^{2}}\right) F_{ \pm s}(\omega, x)=\Delta(x)
$$

in the $\mathrm{S}$ layers and

$$
\begin{aligned}
(\omega & \left.-\frac{D_{f}}{2} \frac{\partial^{2}}{\partial x^{2}} \pm i h+\frac{1}{\tau_{z}}+\frac{2}{\tau_{x}}\right) F_{ \pm f}(\omega, x)+\left(\frac{1}{\tau_{s o}}-\frac{1}{\tau_{x}}\right) \\
& \times\left[F_{ \pm f}(\omega, x)-F_{\mp f}(\omega, x)\right]=0
\end{aligned}
$$

in the $\mathrm{F}$ layers, where $\Delta(x)$ is the superconducting order parameter, $h$ is the exchange field, $D_{s}\left(D_{f}\right)$ is the diffusion coefficient in the $\mathrm{S}(\mathrm{F})$ layer and $\omega$ are the Matsubara frequencies, $\omega=2 \pi T\left(n+\frac{1}{2}\right)$. The parameter $\tau_{s o}$ is the spin-orbit scattering time. The magnetic scattering times are $\tau_{z}$ $=\tau_{2} S^{2} /\left\langle S_{z}^{2}\right\rangle$ and $\tau_{x}=\tau_{2} S^{2} /\left\langle S_{x}^{2}\right\rangle$. The rate $\tau_{2}^{-1}$ is proportional to the square of the exchange interaction potential (the notations are the same as in Ref. 10). Note that the microscopic Green function theory of superconductors with magnetic impurities and spin-orbit scattering was proposed by Abrikosov and Gor'kov. ${ }^{11}$

In addition, the usually used ferromagnets present a strong uniaxial anisotropy. In that case, the perpendicular fluctuations of the exchange field are suppressed, that is, $\tau_{x}^{-1} \sim 0$. Therefore, henceforth, $\tau_{z}$ will be denoted as the magnetic scattering time $\tau_{m}$. The spin-flip scattering is now simply incorporated by replacing $\omega$ by $\omega+1 / \tau_{m}$ in the standard Usadel equation, where $\tau_{m}$ is the magnetic scattering time (see, for example, Ref. 12). Note that the Usadel equations in the F layers are not coupled any more when $\tau_{s o}^{-1}=0$, and only one equation is needed.

\section{THEORETICAL DESCRIPTION OF THE NONMONOTONIC DEPENDENCE OF $T_{c}^{*}$}

The common feature of all the S/F bilayered and multilayered heterostructures is the nonmonotonic evolution of the critical temperature $T_{c}^{*}$ with the thickness of the ferromagnetic layer. This behavior was first predicted by Buzdin and Kuprianov ${ }^{13}$ and Radovic et al. ${ }^{14}$ and was since then intensively studied both theoretically and experimentally (as a review, see Ref. 1). The presence of magnetic scattering can result in an additional decrease of the transition temperature (on the contrary, nonmagnetic impurities do not affect the transition temperature). Note that the theoretical description of $T_{c}^{*}$ for ferromagnetic layers with spin-orbit scattering was performed by Demler et al. ${ }^{15}$ and Oh et al. ${ }^{16}$ In the present section, we therefore mainly focus on the influence of spinflip process and neglect spin-orbit scattering. We report here on the influence of the spin-flip scattering on the nonmonotonic dependence of $T_{c}^{*}$ when the thickness of the superconducting layer is supposed to be small, i.e., $d_{s} \ll \xi_{s}$. In that case, an analytical solution may be obtained. It should also be underlined that the question of the spin-flip role was first addressed by Tagirov ${ }^{17}$ in the discussion of $T_{c}^{*}$ of SF multi-

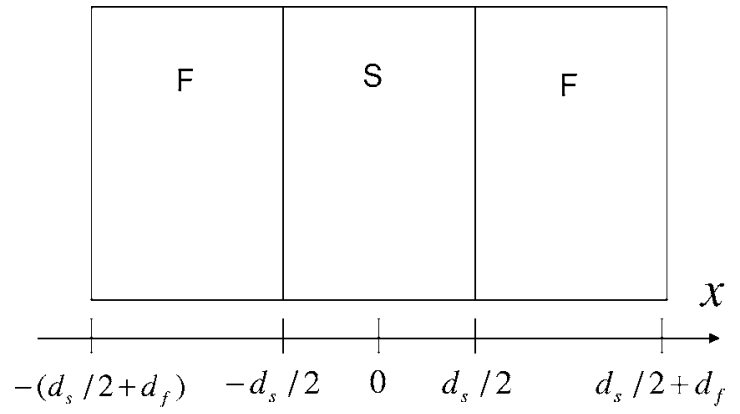

FIG. 1. Geometry of the considered system.

layers and by Fal'ko et al. ${ }^{18}$ in the study of the resistance of a diffusive ferromagnet/normal junction.

\section{A. Influence of spin-flip scattering on $T_{c}^{*}$}

We consider a S/F multilayered system with $\mathrm{F}$ layers of thickness $d_{f}$ and parallel magnetization directions and S layers of thickness $d_{s}$ (see Fig. 1) (this system is also equivalent to a $\mathrm{S} / \mathrm{F}$ bilayer of thicknesses $d_{f} / 2$ and $d_{s} / 2$, respectively). Further, we assume that the SF interfaces are not magnetically active, i.e., there is no rotation of the quasiparticle spin at the interfaces as considered in Refs. 19-22. Under these conditions, long-range spin-triplet superconductivity does not appear. ${ }^{3}$ Discussion of the role of spin-flip scattering in a ferromagnet in combination with magnetically active SF interfaces and/or noncollinear magnetizations requires separate study.

The anomalous Green function $F_{s}$ varies a little in the $\mathrm{S}$ layer and may be approximated by a simple expansion up to the second order $x^{2}$ (see, for example, Refs. 23 and 24)

$$
F_{s}(x, \omega)=F_{0}\left(1-\frac{\beta_{\omega}}{2} x^{2}\right),
$$

where $F_{0}$ is the value of the anomalous Green function at the center of the $\mathrm{S}$ layer. Moreover, in that case, the spatial variation of the pair potential $\Delta(x)$ can be neglected, $\Delta(x) \sim \Delta$. It follows from Eq. (1) that $F_{0}=\Delta /\left(\omega+\tau_{s}^{-1}\right)$ where $\tau_{s}^{-1}$ $=\left(D_{s} / 2\right) \beta_{\omega}$ is the complex pair-breaking parameter.

The Usadel equation in the $\mathrm{S}$ layer is completed by the self-consistency equation

$$
\Delta(x) \ln \frac{T_{c}}{T_{c}^{*}}+\pi T_{c}^{*} \sum_{\omega}\left(\frac{\Delta(x)}{|\omega|}-F_{ \pm s}(x, \omega)\right)=0,
$$

where $T_{c}$ is the bare transition temperature of the superconducting layer in the absence of the proximity effect. Hence, this self-consistent equation gives for $T_{c}^{*}$

$$
\ln \frac{T_{c}^{*}}{T_{c}}=\Psi\left(\frac{1}{2}\right)-\operatorname{Re} \Psi\left(\frac{1}{2}+\frac{1}{2 \pi T_{c}^{*} \tau_{s}}\right) .
$$

If the temperature variation is small $\left(\tau_{s}^{-1} \ll T_{c}\right)$, Eq. (5) becomes

$$
\frac{T_{c}-T_{c}^{*}}{T_{c}}=\frac{\pi}{4 T_{c}} \operatorname{Re}\left(\tau_{s}^{-1}\right) .
$$


The boundary conditions for the linearized Usadel equation $\operatorname{are}^{25}$

$$
\begin{gathered}
\left(\frac{\partial F_{s}}{\partial x}\right)_{ \pm d_{s} / 2}=\frac{\sigma_{n}}{\sigma_{s}}\left(\frac{\partial F_{f}}{\partial x}\right)_{ \pm d_{s} / 2}, \\
F_{s}\left(d_{s} / 2\right)=F_{f}\left(d_{s} / 2\right)-\xi_{n} \gamma_{B}\left(\frac{\partial F_{f}}{\partial x}\right)_{d_{s} / 2}, \\
F_{s}\left(-d_{s} / 2\right)=F_{f}\left(-d_{s} / 2\right)+\xi_{n} \gamma_{B}\left(\frac{\partial F_{f}}{\partial x}\right)_{-d_{s} / 2}
\end{gathered}
$$

with $\sigma_{n}\left(\sigma_{s}\right)$ the conductivity of the $\mathrm{F}(\mathrm{S})$ layer, $\xi_{n}$ $=\sqrt{D_{f} / 2 \pi T_{c}}$, and $\gamma_{B}=R_{b} \sigma_{n} / \xi_{n}$ the interface transparency, related to the $\mathrm{S} / \mathrm{F}$ resistance per unit area $R_{b}$. They lead to the determination of the pair-breaking parameter $\tau_{s}^{-1}$,

$$
\tau_{s}^{-1}=-\frac{D_{s}}{d_{s}} \frac{\sigma_{n}}{\sigma_{s}} \frac{F_{f}^{\prime}\left(d_{s} / 2\right) / F_{f}\left(d_{s} / 2\right)}{1-\xi_{n} \gamma_{B} F_{f}^{\prime}\left(d_{s} / 2\right) / F_{f}\left(d_{s} / 2\right)} .
$$

Next, the resolution of the Usadel equation in the F layers (2) with symmetry consideration gives rise to the expression of the anomalous Green function in the F layer

$$
F_{f}(x, \omega>0)=A \cosh \left[k\left(x-d_{s} / 2-d_{f} / 2\right)\right]
$$

in the 0 phase and

$$
F_{f}(x, \omega>0)=B \sinh \left[k\left(x-d_{s} / 2-d_{f} / 2\right)\right]
$$

in the $\pi$ phase. If $T_{c}<\tau_{m}^{-1}, h$, we may neglect the Matsubara frequencies in the expression for $k$ that becomes

$$
k=\frac{\sqrt{2}}{\xi_{f}} \sqrt{i+\alpha}=\frac{1}{\xi_{f 1}}+i \frac{1}{\xi_{f 2}},
$$

with $\xi_{f}=\sqrt{D_{f} / h}$ and $\alpha=1 / \tau_{m} h$. The two parameters $\xi_{f 1}$ and $\xi_{f 2}$ are, respectively, the decay characteristic length and the oscillation period, and may be written as

$$
\begin{gathered}
\xi_{f 1}=\frac{\xi_{f}}{\sqrt{\sqrt{1+\alpha^{2}}+\alpha}}, \\
\xi_{f 2}=\frac{\xi_{f}}{\sqrt{\sqrt{1+\alpha^{2}}-\alpha}} .
\end{gathered}
$$

For $\tau_{m}^{-1}=0$, Eqs. (13) and (14) reduce to $\xi_{f 1}=\xi_{f 2}=\xi_{f}$. As expected, it is found that the decay length and oscillation period are the same in absence of spin-flip scattering.

The pair-breaking parameter may be determined and it reads

$$
\tau_{s, 0}^{-1}(\omega>0)=\tau_{0}^{-1} \frac{q \tanh \left(q \widetilde{d}_{f} / 2\right)}{1+\tilde{\gamma} q \tanh \left(q \widetilde{d}_{f} / 2\right)}
$$

in the 0 phase and

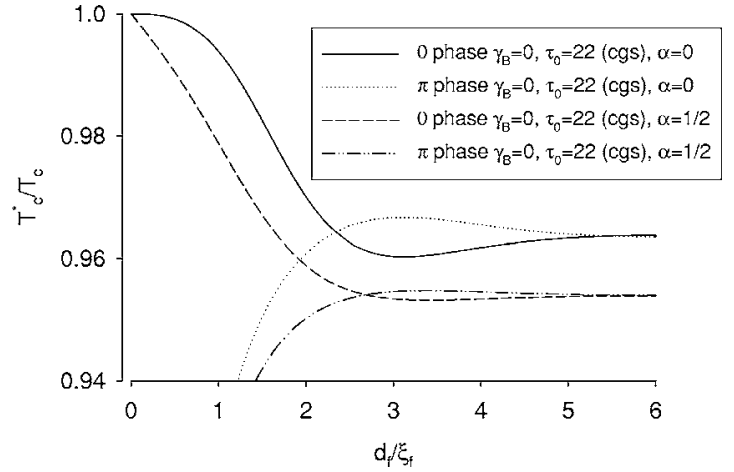

FIG. 2. Influence of the spin-flip scattering on the evolution of critical temperature as a function of the ferromagnetic layer thickness.

$$
\tau_{s, \pi}^{-1}(\omega>0)=\tau_{0}^{-1} \frac{q \operatorname{coth}\left(q \widetilde{d_{f}} / 2\right)}{1+\tilde{\gamma} q \operatorname{coth}\left(q \widetilde{d_{f}} / 2\right)}
$$

in the $\pi$ phase, where $\tau_{0}^{-1}=D_{s} / d_{s} \sigma_{n} / \sigma_{s} 1 / \xi_{f}, \quad \tilde{\gamma}=\left(\xi_{n} / \xi_{f}\right) \gamma_{B}$, $q=k \xi_{f}$, and $\widetilde{d}_{f}=d_{f} / \xi_{f}$.

First, if the interface is supposed to be transparent, analytical expressions of the variation of the temperature may be found if $\left(T_{c}-T_{c}^{*}\right) / T_{c} \ll 1$ :

$$
\frac{4 \tau_{0}}{\pi}\left(T_{c}-T_{c}^{* 0}\right)=\frac{1}{2} \frac{a \sinh \left(a \widetilde{d}_{f}\right)-b \sin \left(a \widetilde{d}_{f}\right)}{\cosh ^{2}\left(a \widetilde{d}_{f}\right) \cos ^{2}\left(b \widetilde{d}_{f}\right)+\sin ^{2}\left(b \widetilde{d}_{f}\right) \sinh ^{2}\left(a \widetilde{d}_{f}\right)},
$$

$$
\frac{4 \tau_{0}}{\pi}\left(T_{c}-T_{c}^{* \pi}\right)=\frac{1}{2} \frac{a \sinh \left(a \widetilde{d}_{f}\right)+b \sin \left(b \widetilde{d}_{f}\right)}{\cosh ^{2}\left(a \widetilde{d}_{f}\right) \sin ^{2}\left(b \widetilde{d}_{f}\right)+\cos ^{2}\left(b \widetilde{d}_{f}\right) \sinh ^{2}\left(a \widetilde{d}_{f}\right)},
$$

where two dimensionless parameters have been introduced, namely, $a=\xi_{f} / \xi_{f 1}=\sqrt{\sqrt{1+\alpha^{2}}+\alpha}$ and $b=\xi_{f} / \xi_{f 2}=\sqrt{\sqrt{1+\alpha^{2}}-\alpha}$, so that $q=a+i b$.

In the general case, however, a numerical analysis has to be performed.

The ratio of the characteristic lengths

$$
\frac{\xi_{f 1}}{\xi_{f 2}}=\frac{\sqrt{\sqrt{1+\alpha^{2}}-\alpha}}{\sqrt{\sqrt{1+\alpha^{2}}+\alpha}}
$$

clearly shows that magnetic scattering decreases the decay length and increases the oscillation period. If $\tau_{m}^{-1} \gg h, \xi_{f 1}$ can become much smaller than $\xi_{f 2}$. In addition, the decrease of $\xi_{f 1}$ makes the observation of the oscillations more difficult.

The evolution of the critical temperature without and with spin-flip scattering is given in Fig. 2. The phase that really occurs is the one with higher critical temperature. It is seen that $\xi_{f 1}$ decreases in the presence of spin flip while $\xi_{f 2}$ increases.

B. Influence of the interface transparency parameter $\gamma_{B}$ on $T_{c}^{*}$

The influence of the interface transparency parameter $\gamma_{B}$ on $T_{c}^{*}$ of SF bilayers was studied before by Aarts et al., ${ }^{26}$ 


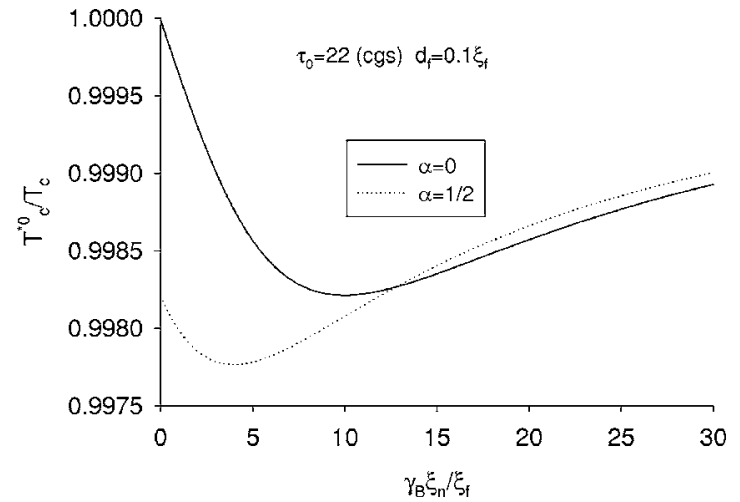

FIG. 3. Evolution of the critical temperature versus the interface transparency parameter for a S/F bilayer.

Fominov et al.,${ }^{27}$ and Tagirov. ${ }^{17}$ Here we extend this discussion taking into account spin-flip scattering in the F layer and considering a broader range of interface transparencies, and demonstrate that additional effects take place in this situation.

The intriguing evolution of $T_{c}^{* 0}$ with the interface transparency parameter $\tilde{\gamma}$ must be underlined (see Fig. 3). If $\alpha$ $=0$, there is no magnetic scattering and it could intuitively be believed that the higher the barrier, the less is the influence of the proximity effect on the $\mathrm{S}$ layer and therefore $T_{c}^{*}(\tilde{\gamma}$ $\gg 1)>T_{c}^{*}(\tilde{\gamma} \sim 1)$. However, it can be seen from Fig. 3 that the critical temperature is a decreasing function of the interface transparency parameter $\tilde{\gamma}$ for a small thickness of the $\mathrm{F}$ layer. This counterintuitive behavior can be qualitatively understood for a S/F bilayer. The probability for a Cooper pair to leave the $\mathrm{S}$ layer is smaller for a low transparent interface $(\tilde{\gamma} \gg 1)$. Nevertheless, the probability for this pair to come back again in the $S$ layer is much higher for a transparent interface. Indeed, when the F layer is thin enough, the reflection of the Cooper pair at the other interface of the $\mathrm{F}$ layer allows the pair to cross the first interface again, which is easier when $\tilde{\gamma}$ is small. Consequently, the staying time in the $F$ layer increases with the increase of the barrier, and when this time becomes higher than the coherence time of the Cooper pair, the pair is destroyed, leading to a weakened superconductivity. Therefore, the critical temperature decreases with increasing barrier in that case. On the other hand, if $\widetilde{d}_{f}$ increases, the Cooper pair is hardly reflected by the external interface of the F layer whatever is the value of $\tilde{\gamma}$ and the critical temperature is expected to increase with increasing barrier.

In addition, an analytical approach using formula (6) leads to the determination of the critical temperature when $\widetilde{d_{f}}<1$. In that case, the pair-breaking parameter in the 0 phase becomes

$$
\operatorname{Re}\left(\tau_{m}^{-1}\right)=\tau_{0}^{-1} \frac{\tilde{\gamma}+\widetilde{d}_{f} / 6}{\left(\tilde{\gamma}+\widetilde{d}_{f} / 6\right)^{2}+\left(1 / \widetilde{d}_{f}\right)^{2}} .
$$

Two behaviors emerge depending on whether $\tilde{\gamma} \widetilde{d}_{f} \gg 1$ or not. Indeed, if $\tilde{\gamma}>\widetilde{d}_{f}^{-1}$, the critical temperature evolution is described by

$$
\left(\frac{T_{c}^{*}}{T_{c}}\right)_{\tilde{\gamma} d_{f}>1}=1-\frac{\pi}{4 T_{c}} \frac{\tau_{0}^{-1}}{\tilde{\gamma}},
$$

which is an increasing function of $\tilde{\gamma}$. On the contrary, if $\tilde{\gamma}<\widetilde{d}_{f}^{-1}$, the critical temperature decreases when $\tilde{\gamma}$ increases, following

$$
\left(\frac{T_{c}^{*}}{T_{c}}\right)_{\tilde{\gamma}_{f}<1}=1-\frac{\pi}{4 T_{c}} \widetilde{d}_{f}^{2} \tau_{0}^{-1} \tilde{\gamma} .
$$

The critical temperature evolution with $\tilde{\gamma}$ presented in Fig. 3 is therefore understood.

In presence of small magnetic scattering, the critical temperature evolution remains qualitatively the same. As shown in Fig. 3, it appears that superconductivity may be less destroyed when there is spin-flip scattering. In that case, the pair-breaking parameter becomes

$$
\operatorname{Re}\left(\tau_{m}^{-1}\right)=\tau_{0}^{-1} \frac{\tilde{\gamma}+\alpha / \widetilde{d}_{f}+\widetilde{d}_{f} / 6}{\left(\tilde{\gamma}+\alpha / \widetilde{d}_{f}+\widetilde{d}_{f} / 6\right)^{2}+\left(1 / \widetilde{d}_{f}\right)^{2}} .
$$

The critical temperature is described by

$$
\left(\frac{T_{c}^{*}}{T_{c}}\right)_{\tilde{\gamma} \tilde{d}_{f}>1}=\left(\frac{T_{c}^{*}(\alpha=0)}{T_{c}}\right)_{\tilde{\gamma} \tilde{d}_{f}>1}+\frac{\pi}{4 T_{c}} \frac{\tau_{0}^{-1}}{\tilde{\gamma}} \frac{\alpha}{\widetilde{d}_{f} \tilde{\gamma}},
$$

when $\widetilde{d}_{f} \tilde{\gamma}>1$, and magnetic scattering leads to a slight enhancement of the transition temperature. On the contrary, if $\tilde{\gamma} d_{f}<1$,

$$
\left(\frac{T_{c}^{*}}{T_{c}}\right)_{\tilde{\gamma}_{f}<1}=\left(\frac{T_{c}^{*}(\alpha=0)}{T_{c}}\right)_{\tilde{\gamma} \tilde{d}_{f}<1}-\frac{\pi}{T_{c}} \widetilde{d}_{f} \tau_{0}^{-1} \alpha,
$$

and spin flip implies the decrease of the transition temperature.

\section{Influence of spin-orbit scattering and "perpendicular" spin flip}

Let us now consider briefly the general case, with spinorbit and/or perpendicular spin-flip scattering. An additional parameter has to be introduced, namely,

$$
\alpha_{\perp}=\frac{1}{h}\left(\frac{1}{\tau_{x}}-\frac{1}{\tau_{s o}}\right),
$$

and the parameter $\alpha$ now becomes

$$
\alpha=\frac{1}{h}\left(\frac{1}{\tau_{z}}+\frac{2}{\tau_{x}}\right) .
$$

In that case, expressions (15) and (16) are modified. In the 0 phase, $q \tanh \left(q d_{f} / 2\right)$ is replaced by

$$
\begin{aligned}
& q \tanh \left(q \widetilde{d_{f}} / 2\right) \\
& +\beta \frac{q^{*} \tanh \left(q^{*} \widetilde{d}_{f} / 2\right)-q \tanh \left(q \widetilde{d}_{f} / 2\right)}{\beta+\left[1+\tilde{\gamma}^{*} \tanh \left(q^{*} \widetilde{d}_{f} / 2\right)\right] /\left[1+\tilde{\gamma} q \tanh \left(q \tilde{d}_{f} / 2\right)\right]},
\end{aligned}
$$

where $q$ becomes 


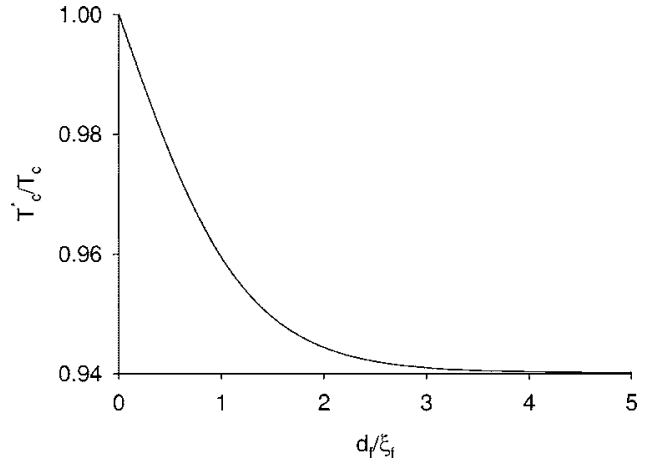

FIG. 4. Monotonic evolution of the critical temperature for a transparent interface, and for $\tau_{0}=22$ (cgs), $\alpha=1$, and $\alpha_{\perp}=-3 / 2$.

$$
q^{2}=2\left(\frac{\omega}{h}+\alpha-\alpha_{\perp}+i \sqrt{1-\alpha_{\perp}^{2}}\right)
$$

and $\beta$ is

$$
\beta=-\alpha_{\perp} \frac{\alpha_{\perp}-i \sqrt{1-\alpha_{\perp}^{2}}}{1+\sqrt{1-\alpha_{\perp}^{2}}} .
$$

In the $\pi$ phase, $q \operatorname{coth}\left(q \widetilde{d_{f}} / 2\right)$ is replaced by

$$
\begin{aligned}
& q \operatorname{coth}\left(q \widetilde{d}_{f} / 2\right) \\
& +\beta \frac{q^{*} \operatorname{coth}\left(q^{*} \widetilde{d_{f}} / 2\right)-q \operatorname{coth}\left(q \tilde{d}_{f} / 2\right)}{\beta+\left[1+\tilde{\gamma} q^{*} \operatorname{coth}\left(q^{*} \widetilde{d}_{f} / 2\right)\right] /\left[1+\tilde{\gamma} q \operatorname{coth}\left(q \tilde{d}_{f} / 2\right)\right]} .
\end{aligned}
$$

Therefore, the influence of "perpendicular" spin-flip scattering and spin-orbit scattering is quite similar to the influence of "parallel" spin-flip processes, in the sense that it also implies the decrease of the decaying length and the increase of the oscillation period. However, a special situation arises when $\alpha_{\perp}>1$. Then, the oscillations of the Cooper pair wave function are completely destroyed. A similar conclusion for spin-orbit mechanism was obtained in Ref. 15. In fact, the influence of the perpendicular magnetic scattering is analogous to the spin-orbit scattering (see Fig. 4). Probably the role of perpendicular spin-flip or spin-orbit scattering is important for the understanding of experimental results where no oscillation of the critical temperature was detected. Besides, note that the critical temperature oscillations cannot disappear when there is only parallel spin-flip scattering. Interestingly, as it follows from Eq. (26), the perpendicular magnetic scattering can compensate the spin-orbit scattering and restore the oscillatory behavior of the Cooper pair wave function, which in turn leads to the nonmonotonic dependence of $T_{c}^{*}\left(d_{f}\right)$.

\section{BEHAVIOR OF THE CRITICAL CURRENT $I_{c}$}

A Josephson junction may be realized with $\mathrm{S} / \mathrm{F} / \mathrm{S}$ sandwiches in which the weak link between the two superconductors is ensured by the ferromagnetic layer. The supercurrent $J_{s}(\varphi)$ flowing across the structure can be expressed as $J_{s}(\varphi)=I_{c} \sin (\varphi)$, where $I_{c}$ is the critical current and $\varphi$ stands for the phase difference between the two superconducting layers. A standard junction has at equilibrium $I_{c}>0$ and $\varphi$ $=0$, and therefore, no current exists. It may appear, however, that $I_{c}$ becomes negative, which implies that the equilibrium phase difference is $\varphi=\pi$ and the ground state undergoes a $\pi$ phase shift. This so-called $\pi$ junction was first predicted for $\mathrm{S} / \mathrm{F} / \mathrm{S}$ structures in the clean limit, ${ }^{28}$ and later in the more realistic case of the diffusive limit. ${ }^{29}$ The critical current $I_{c}$ in $\mathrm{S} / \mathrm{F} / \mathrm{S}$ junctions is controlled by the exchange field in the ferromagnet, the interface transparency parameter $\gamma_{B}$, and the spin-flip and spin-orbit scattering rates. In this section we will discuss the influence of these parameters on $I_{c}$, paying the main attention to the role of spin-orbit and magnetic scatterings. In addition, note that the spin-orbit effect on the critical current has already been studied for $\mathrm{S} / \mathrm{F}$ bilayers separated by an insulator with thin $\mathrm{F}$ layers ${ }^{30}$ and for the FSFSF structure with noncollinear magnetizations of $\mathrm{F}$ layers. ${ }^{31}$ We will consider a symmetric $S / F / S$ structure with the $\mathrm{F}$ layer having thickness $d_{f}$ and assume that the dirty limit conditions are satisfied for $\mathrm{S}$ and $\mathrm{F}$ materials. We will assume that the rigid boundary condition $\gamma_{B}$ $\gg \min \left\{\sigma_{n} \xi_{S} / \sigma_{s} \xi_{n}, 1\right\}$ is satisfied, when the suppression of superconductivity in $\mathrm{S}$ can be neglected and the linearized Usadel equations (2) can be used. The case of transparent interfaces, $\gamma_{B}=0$, will be considered at the end.

\section{A. General expression for $I_{c}$}

To derive the general expression for $I_{c}$ we should supplement (2) by the boundary conditions ${ }^{25}$ at S/F interfaces $(x$ $\left.= \pm d_{f}\right)$

$$
\begin{gathered}
\gamma_{B} \xi_{n} \frac{\partial}{\partial x} F_{ \pm f}\left( \pm d_{f}\right)= \pm G_{S}\left(\frac{\Delta\left( \pm d_{f}\right)}{\omega}-F_{ \pm f}\left( \pm d_{f}\right)\right) \operatorname{sgn}(\omega), \\
G_{S}=\frac{\omega}{\sqrt{\omega^{2}+|\Delta|^{2}}}, \quad \Delta\left( \pm d_{f}\right)=|\Delta| \exp ( \pm i \varphi / 2) .
\end{gathered}
$$

The solution of the boundary problem (2), (32) has the form

$$
\begin{aligned}
F_{+f}= & \frac{1}{2}\left\{\left(1+\eta_{\omega}\right)\left[A_{\omega+} \cosh \left(k_{+} x\right)+B_{\omega+} \sinh \left(k_{+} x\right)\right]+\left(1-\eta_{\omega}\right)\right. \\
& \left.\times\left[A_{\omega-} \cosh \left(k_{-} x\right)+B_{\omega-} \sinh \left(k_{-} x\right)\right]\right\}, \\
F_{-f}= & \frac{1}{2}\left\{\left(1-\eta_{\omega}\right)\left[A_{\omega+} \cosh \left(k_{+} x\right)+B_{\omega+} \sinh \left(k_{+} x\right)\right]-\left(1+\eta_{\omega}\right)\right. \\
& \left.\times\left[A_{\omega-} \cosh \left(k_{-} x\right)+B_{\omega-} \sinh \left(k_{-} x\right)\right]\right\},
\end{aligned}
$$

where the coefficients $A_{\omega \pm}$ and $B_{\omega \pm}$ are given in the Appendix, while

$$
\eta_{\omega}=\frac{\alpha_{s o}+\sqrt{\alpha_{s o}^{2}-1}}{i h \operatorname{sgn}(\omega)}, \quad k_{ \pm}=\frac{\sqrt{2}}{\xi_{f}} \sqrt{|\widetilde{\omega}|+\alpha_{s o}+\alpha \pm \sqrt{\alpha_{s o}^{2}-1}} .
$$

The parameter $\alpha_{s o}$ is defined by $\alpha_{s o}=1 /\left(h \tau_{s o}\right), \alpha=1 /\left(h \tau_{m}\right)$, and $\widetilde{\omega}=\omega / h$. Note that in the general case perpendicular spin flip has to be added in $\alpha_{s o}$ and $\alpha$. The general expression for the supercurrent is 


$$
J_{S}=\frac{i \pi T \sigma_{n}}{4 e} \sum_{\omega=-\infty, \sigma= \pm}^{\infty}\left(\tilde{F}_{\sigma f} \frac{\partial}{\partial x} F_{\sigma f}-F_{\sigma f} \frac{\partial}{\partial x} \tilde{F}_{\sigma f}\right)
$$

where $\widetilde{F}_{ \pm f}(x, \omega)=F_{ \pm f}^{*}(x,-\omega)$. Substituting Eqs. (33) and (34) into the above expression and taking into account the symmetry relations (given in the Appendix), we get $J_{S}=I_{c} \sin \varphi$.

For large spin-orbit scattering, i.e., $\tau_{s o}^{-1} \geqslant h$, the critical current $I_{c}$ equals

$$
\frac{e \xi_{n} I_{c}}{2 \pi T_{c} \sigma_{n}}=\frac{T}{T_{c n=0}} \sum^{\infty} \frac{\Delta^{2} G_{S}^{2}}{\omega^{2}\left(1+\eta_{\omega}^{2}\right)}\left(\kappa_{+}+\eta_{\omega}^{2} \kappa_{-}\right),
$$

where

$$
\kappa_{ \pm}=\frac{\xi_{n} k_{ \pm}}{\left(\gamma_{B}^{2} \xi_{n}^{2} k_{ \pm}^{2}+G_{S}^{2}\right) \sinh \left(k_{ \pm} d_{f}\right)+2 G_{S} \gamma_{B} \xi_{n} k_{ \pm} \cosh \left(k_{ \pm} d_{f}\right)} .
$$

At larger exchange field $h \geqslant \tau_{s o}^{-1}$, the critical current becomes

$$
\begin{aligned}
& \frac{e I_{c}}{2 \pi T_{c} \sigma_{n}}=\frac{T}{\xi_{f} T_{c}} \sum_{n=0}^{\infty} \frac{\Delta^{2} G_{S}^{2}}{\omega^{2}} \\
& \quad \times \operatorname{Re}\left(\frac{(a+i b)\left(1+i 2 / \sqrt{\alpha_{s o}^{-2}-1}\right)}{\left[\left(\gamma_{B} \xi_{n} k\right)^{2}+G_{S}^{2}\right] \sinh \left(k d_{f}\right)+2 G_{S} \gamma_{B} \xi_{n} k \cosh \left(k d_{f}\right)}\right),
\end{aligned}
$$

where $k=q / \xi_{f}$ and $q=a+i b$, with

$$
a=\sqrt{|\widetilde{\omega}|+\alpha_{s o}+\alpha+\sqrt{(|\widetilde{\omega}|+\alpha)^{2}+2(|\widetilde{\omega}|+\alpha) \alpha_{s o}+1}},
$$

$$
b=\sqrt{\frac{1-\alpha_{s o}^{2}}{|\widetilde{\omega}|+\alpha_{s o}+\alpha+\sqrt{(|\widetilde{\omega}|+\alpha)^{2}+2(|\widetilde{\omega}|+\alpha) \alpha_{s o}+1}}} .
$$

In appropriate limits, expressions (37) and (39) transform into the previously obtained results. ${ }^{1,15,25,24,32}$ This approach is valid if $F_{\omega \pm} \ll 1$ or

$$
\gamma_{B} \gg \gamma, \quad \frac{a}{a^{2}+b^{2}} \frac{\Delta}{\pi T_{c}} \frac{1}{\min \left\{1, d_{f} / \xi_{n}\right\}} .
$$

As follows from Eqs. (39)-(41), in the case of strong spin-orbit scattering $\tau_{s o}^{-1} \geqslant h$, the critical current decays monotonically with the increase of $d_{f}$ with two decay lengths $k_{ \pm}^{-1}$ defined by Eq. (35). It is seen from (35) that in the limit $h \rightarrow 0$, the parameter $\eta_{\omega} \rightarrow \infty$. As a result, the contribution to the critical current in Eq. (37) comes only from the $\kappa_{-}$component with the length scale $k_{-}^{-1}$ which describes the case of a S/N/S junction in which spin-orbit scattering does not influence $I_{c}$. With the increase of $h$, the contribution to $I_{c}$ from the faster decaying $\kappa_{+}$component $\left(k_{+}>k_{-}\right)$also increases and the difference between $k_{+}$and $k_{-}$decreases. Finally, when $h=\tau_{s o}^{-1}$, both scales coincide, $k_{+}=k_{-}$, and the components $\kappa_{+}, \kappa_{-}$provide equal contributions to $I_{c}$.

For relatively weak spin-orbit scattering $\tau_{s o}^{-1} \leqslant h$, the dependence $I_{c}\left(d_{f}\right)$ follows the damped oscillation law, when two length scales $\xi_{f 1}, \xi_{f 2}$ can be introduced describing, respectively, the decay and the oscillation period of $I_{c}\left(d_{f}\right)$. In this section, we will concentrate on the case $\tau_{s o}^{-1} \leqslant h$. The scales $\xi_{f 1}, \xi_{f 2}$ are related to $a, b$ and will be discussed in detail below in different limits.

\section{Limit of small F-layer thickness and large $\gamma_{B}$}

In the limit of small thickness $d_{f} \ll \xi_{f 1}$, and large interface transparency parameter $\gamma_{B} \gg d_{f} / \xi_{n},\left(\pi T_{c} G_{S} \xi_{n}\right) /\left(d_{f}\left(\omega+\tau_{m}^{-1}\right.\right.$ $+\tau_{s 0}^{-1}$ ) we can neglect the terms $G_{S}^{2}$ and $G_{S} \gamma_{B} \xi_{n} k\left(k d_{f}\right)^{2}$ in the denominator of Eq. (39) and with accuracy of better than $\left(d_{f} / \xi_{f 1}\right)^{3}$ get

$$
\begin{aligned}
\frac{e R_{N} I_{c}}{4 \pi T_{c}}= & \frac{T}{\tilde{\gamma} d_{f} T_{c}} \xi_{f} \sum_{n=0}^{\infty} \frac{\Delta^{2} G_{S}^{2}}{\omega^{2}} \\
& \times\left(\frac{\Omega_{1}+2 \alpha_{s o}+\left(\Omega^{2}-v^{2}+4 \alpha_{s o} \Omega\right) d_{f}^{2} / 6 \xi_{f}^{2}}{\Omega_{1}^{2}+v^{2}+\left(d_{f}^{2} / 3 \xi_{f}^{2}\right) \Omega\left(\Omega^{2}+v^{2}\right)}\right),
\end{aligned}
$$

where $R_{N}=2 R_{B}$ is the normal junction resistance, $\tilde{\gamma}$ $=\gamma_{B} \xi_{n} / \xi_{f}, \widetilde{\omega}=\omega / h$, and

$$
\Omega=\widetilde{\omega}+\alpha_{s o}+\alpha, \quad v^{2}=1-\alpha_{s o}^{2}, \quad \Omega_{1}=\Omega+2 G_{S} \frac{\pi T_{c} \xi_{n}}{\gamma_{B} h d_{f}} .
$$

If additionally

$$
\gamma_{B} \gg \frac{6 \xi_{n}^{3} \pi T_{c}}{d_{f}^{3}\left[\left(\pi T_{c} / h+\alpha_{s o}+\alpha\right)^{2}+1-\alpha_{s o}^{2}\right) h}
$$

then

$$
\frac{e R_{N} I_{c}}{4 \pi T_{c}}=\frac{\xi_{f}}{\tilde{\gamma} d_{f}}\left(\frac{T}{T_{c}} \sum_{n=0}^{\infty} \frac{\Delta^{2}}{\omega^{2}+\Delta^{2}} \frac{\Omega+2 \alpha_{s o}}{\left(\Omega^{2}+v^{2}\right)}-\frac{d_{f}^{2}}{6 \xi_{f}^{2}} \frac{\Delta}{2 T_{c}} \tanh \frac{\Delta}{2 T}\right) .
$$

For $h \gg \pi T_{c}, \tau_{m}^{-1}, \tau_{s 0}^{-1}$ and $T \ll T_{c}$, the sum in Eq. (44) can be calculated by transforming from summation into integration over $\omega$ resulting in

$$
\frac{e R_{N} I_{c}}{4 \pi T_{c}}=\frac{\Delta}{2 T_{c}} \frac{\xi_{f}}{\widetilde{\gamma} d_{f}}\left(\frac{2 \Delta}{h \pi} \ln \frac{h}{\Delta}+\frac{3}{h \tau_{s o}}+\frac{1}{h \tau_{m}}-\frac{d_{f}^{2}}{6 \xi_{f}^{2}}\right) .
$$

From Eq. (45) it follows that the transformation from the 0 to $\pi$ junction may occur when the thickness $d_{f}$ exceeds some critical value $d_{\pi}$,

$$
d_{f} \gtrsim d_{\pi}, \quad d_{\pi}=\sqrt{6} \xi_{f} \sqrt{\frac{2 \Delta}{\pi h} \ln \frac{h}{\Delta}+\frac{3}{h \tau_{s o}}+\frac{1}{h \tau_{m}}} .
$$

For $\tau_{s o}^{-1}, \tau_{m}^{-1} \ll h$ this result agrees with Ref. 32. It is interesting to note that in this range of parameters, the condition (46) of the transition to the $\pi$ state does not depend on the properties of the interfaces. As is also seen from Eq. (46), both spin-orbit and spin-flip scattering increase the thickness $d_{\pi}$ corresponding to the first 0 to $\pi$ transition.

If $\tilde{\gamma} d_{f} / \xi_{f} \leqslant 1$, then the term $G_{S} \xi_{f} /\left(\tilde{\gamma} d_{f}\right)$ in (43) is not small. As a result, the transition to a $\pi$ state should depend on the properties of the interfaces and occurs at $h$ larger than the critical value $h_{\pi} \propto \pi T_{c}\left(\xi_{n} / d_{f}\right)$ following from Eq. (45).

Therefore, in the limit of small $d_{f} \ll \xi_{f 1}$ and large $\gamma_{B} \gg 1$ the transition from the 0 to $\pi$ junction exists only if the 
exchange energy $h \geqslant h_{\pi} \propto \pi T_{c}\left(\xi_{n} / d_{f}\right)$ sufficiently exceeds $\pi T_{c}$. The smaller the interface transparency parameter $\gamma_{B}$, the larger should be the exchange energy $h$. The possibility of the $0-\pi$ transition in junctions with small thickness $d_{f}$ in the case of large $\gamma_{B}$ is related to the multiple scattering at the boundaries. As a result, the electrons spend more time in the F layer and its influence on superconductivity is enhanced. This is the manifestation of the same mechanism that leads in $\mathrm{S} / \mathrm{F}$ bilayers to the critical temperature decrease with the increase of the interface transparency parameter (see Sec. IIB).

\section{Limit of large F-layer thickness}

In the limit of large $d_{f} \gg \xi_{f 1}$ for the critical current from Eqs. (39)-(41), we have

$$
\begin{aligned}
\frac{e I_{c}}{2 \pi T_{c} \sigma_{n}}= & \frac{2 T}{\xi_{f} T_{c}} \sum_{n=0}^{\infty} \frac{\Delta^{2} G_{S}^{2}}{\omega^{2}} \frac{u \sin \left(b d_{f} / \xi_{f}\right)+v \cos \left(b d_{f} / \xi_{f}\right)}{\left[\left(G_{S}+a \tilde{\gamma}\right)^{2}+\tilde{\gamma}^{2} b^{2}\right]^{2}} \\
& \times \exp \left(-a \frac{d_{f}}{\xi_{f}}\right),
\end{aligned}
$$

where the coefficients $u$ and $v$ are defined by

$$
\begin{gathered}
u=b\left[G_{S}^{2}-\left(a^{2}+b^{2}\right) \widetilde{\gamma}^{2}\right]+\frac{2}{\sqrt{\alpha_{s o}^{-2}-1}} \\
\times G_{S}^{2}\left[a+\widetilde{\gamma}\left(a^{2}+b^{2}\right)\left(2 G_{S}+a \widetilde{\gamma}\right)\right], \\
v=G_{S}^{2} a+\widetilde{\gamma}\left(a^{2}+b^{2}\right)\left(2 G_{S}+a \tilde{\gamma}\right)-\frac{2}{\sqrt{\alpha_{s o}^{-2}-1}} \\
\times b\left[G_{S}^{2}-\left(a^{2}+b^{2}\right) \widetilde{\gamma}^{2}\right] .
\end{gathered}
$$

If additionally $\min \left\{h, \tau_{s o}^{-1}, \tau_{m}^{-1}\right\} \gg \pi T_{c}$, then both $a$ and $b$ may be considered as independent of Matsubara frequencies, since the sum in (39) converges at $\omega \approx \pi T_{c}$. In this case

$$
\frac{e \xi_{f} I_{c}}{2 \pi T_{c} \sigma_{n}}=\left(\Sigma_{1} \sin \frac{d_{f}}{\xi_{f 2}}+\Sigma_{2} \cos \frac{d_{f}}{\xi_{f 2}}\right) \exp \left(-\frac{d_{f}}{\xi_{f 1}}\right),
$$

where

$$
\begin{aligned}
& \Sigma_{1}=\frac{2 T}{T_{c}} \sum_{n=0}^{\infty} \frac{\Delta^{2} G_{S}^{2}}{\omega^{2}} \frac{u}{\left[\left(G_{S}+a \tilde{\gamma}\right)^{2}+b^{2} \widetilde{\gamma}^{2}\right]^{2}}, \\
& \Sigma_{2}=\frac{2 T}{T_{c}} \sum_{n=0}^{\infty} \frac{\Delta^{2} G_{S}^{2}}{\omega^{2}} \frac{v}{\left[\left(G_{S}+a \tilde{\gamma}\right)^{2}+b^{2} \tilde{\gamma}^{2}\right]^{2}} .
\end{aligned}
$$

The two characteristic length scales are given by the following expressions:

$$
\begin{gathered}
\xi_{f 1}=\xi_{f} \sqrt{\frac{1}{\alpha+\alpha_{s o}+\sqrt{\alpha^{2}+2 \alpha \alpha_{s o}+1}}}, \\
\xi_{f 2}=\xi_{f} \sqrt{\frac{\alpha+\alpha_{s o}+\sqrt{\alpha^{2}+2 \alpha \alpha_{s o}+1}}{1-\alpha_{s o}^{2}}},
\end{gathered}
$$

which generalize Eqs. (13) and (14) for the case of the presence of the spin-orbit scattering in a ferromagnet. One can see that with the increase of both scattering rates $\tau_{m}^{-1}$ and $\tau_{s o}^{-1}$ the decay length $\xi_{f 1}$ decreases, while the oscillation period $\xi_{f 2}$ increases.

For a weak exchange field $h \ll \pi T_{c}$ and sufficiently high temperatures $\pi T \gg\left\{\tau_{s o}^{-1}, \tau_{m}^{-1}, h\right\}$, with $h>\tau_{s o}^{-1}$, only the first term with $n=0$ in Eq. (39) is important and we have

$$
\frac{e \xi_{f} I_{c}}{2 \pi T_{c} \sigma_{n}}=\frac{2 \Delta^{2} G_{0}}{\pi^{2} T T_{c}} \frac{\Sigma_{1} \sin \left(d_{f} / \xi_{f 2}\right)+\Sigma_{2} \cos \left(d_{f} / \xi_{f 2}\right)}{\left[\left(G_{0}+a_{0} \tilde{\gamma}\right)^{2}+b_{0}^{2} \widetilde{\gamma}^{2}\right]^{2}} \exp \left(-\frac{d_{f}}{\xi_{f 1}}\right),
$$

where $G_{0}=\pi T / \sqrt{(\pi T)^{2}+\Delta^{2}}$ and $\Sigma_{1}$ and $\Sigma_{2}$ become

$$
\begin{aligned}
& \Sigma_{1}=\frac{2 T}{T_{c}} \frac{\Delta^{2} G_{0}^{2}}{(\pi T)^{2}} \frac{u_{0}}{\left[\left(G_{0}+a_{0} \tilde{\gamma}\right)^{2}+b_{0}^{2} \tilde{\gamma}^{2}\right]^{2}}, \\
& \Sigma_{2}=\frac{2 T}{T_{c}} \frac{\Delta^{2} G_{0}^{2}}{(\pi T)^{2}} \frac{v_{0}}{\left[\left(G_{0}+a_{0} \tilde{\gamma}\right)^{2}+b_{0}^{2} \widetilde{\gamma}^{2}\right]^{2}} .
\end{aligned}
$$

The parameters $a_{0}, b_{0}, u_{0}$, and $v_{0}$ are obtained replacing $\omega$ by $\pi T$ in expressions (40), (41), and (48). The two characteristic lengths may be written in that case as

$\xi_{f 1}$

$$
=\xi_{f} \sqrt{\frac{1}{\pi T / h+\alpha+\alpha_{s o}+\sqrt{(\pi T / h+\alpha)^{2}+2(\pi T+\alpha) \alpha_{s o}+1}}},
$$

$\xi_{f 2}$

$$
=\xi_{f} \sqrt{\frac{\pi T / h+\alpha+\alpha_{s o}+\sqrt{(\pi T / h+\alpha)^{2}+2(\pi T+\alpha) \alpha_{s o}+1}}{1-\alpha_{s o}^{2}}} .
$$

For $\tau_{s o}^{-1}, \tau_{m}^{-1}=0$, Eqs. (57) and (58) reduce to a simple expression $\xi_{f 1,2}^{1}=\xi_{n}^{1}\left\{\left[h^{2} /\left(\pi T_{c}\right)^{2}+\left(T / T_{c}\right)^{2}\right]^{1 / 2} \pm\left(T / T_{c}\right)\right\}^{1 / 24}$ which describes the temperature variations of both length scales. More precisely, in the considered limits, $\xi_{f 1}^{1} \sim \xi_{n}^{1} \sqrt{2 T / T_{c}}$, while $\xi_{f 2}^{1} \sim \xi_{f 1}^{1} h /\left(2 \pi \sqrt{T T_{c}}\right) \ll \xi_{f 1}^{1}$. One can see that the scattering rates $\tau_{s o}^{-1}, \tau_{m}^{-1}$ make the $T$ variation of $\xi_{f 1,2}$ weaker.

From Eqs. (49) and (54), one can derive the condition for $I_{c}=0$ and obtain

$d_{f n}=d_{f}, \quad \frac{d_{f n}}{\xi_{f 2}}=\pi n-\arctan \left(\frac{\Sigma_{2}}{\Sigma_{1}}\right), \quad n=0,1,2, \ldots$,

when the transitions between 0 and $\pi$ states occur. The position of the first zero, $d_{f 1}$, depends on both the material parameters of the ferromagnetic layer and the properties of the interfaces and superconducting electrodes, while the distance between the zeros is a function only of $\xi_{f 2}$ and therefore depends only on the transport parameters of the ferromagnetic material.

From the structure of coefficients $u$ and $v$ [see Eqs. (48) and (59)], it follows that in the limit of small $\gamma_{B}\left[F_{ \pm f}\right.$ $=\left(\Delta / \sqrt{\omega^{2}+\Delta^{2}}\right) \exp ( \pm i \varphi / 2)$ at $\mathrm{S} / \mathrm{F} \quad$ interfaces $]$ and $\min \left\{h, \tau_{s o}^{-1}, \tau_{m}^{-1}\right\} \gg \pi T_{c}$, 


$$
\frac{d_{f k}}{\xi_{f 1}}=\pi k-\arctan \left(\frac{2 b-a \sqrt{\alpha_{s o}^{-2}-1}}{2 a+b \sqrt{\alpha_{s o}^{-2}-1}}\right), \quad k=1,2, \ldots .
$$

In particular, it follows from Eq. (60) that for small spinorbit and spin-flip scatterings $h \gg \tau_{s o}^{-1}, \tau_{m}^{-1}$,

$$
\frac{d_{f k}}{\xi_{f 1}} \approx \pi k-\frac{\pi}{4}
$$

and the well-known result $d_{f 1} \approx(3 \pi / 4) \xi_{f 2}$ for the first critical thickness for the 0 to $\pi$ state is reproduced. This $d_{f 1}$ value approximately satisfies the condition of validity of the large$d_{f}$ approximation considered in this section.

It follows from Eq. (60) that the critical thickness $d_{f 1}$ increases with $\tau_{m}^{-1}$ and $\tau_{s o}^{-1}$ (see also the numerical results below).

An increase of $\gamma_{B}$ results in the suppression of the magnitude of $F_{ \pm f}\left( \pm d_{f}\right) \sim \Delta\left(1+\gamma_{B}\right)^{-1}$ near S/F interfaces, which leads to the decrease of $d_{f 1}$. Formally it is due to the fact that with an increase of $\gamma_{B}$, the coefficient $b$ in (48) and, hence, $\Sigma_{1}$, may change its sign, resulting in the existence of the solution $d_{f 0}$ of (59) for $n=0$. This solution corresponds to the thickness range in which the simple large- $d_{f}$ approximation (47) is no longer valid. This fact is in full agreement with our consideration performed for the limit of small $d_{f}$; namely, it follows from Eq. (46) that at large $\gamma_{B}$ and $h$ we have

$$
d_{f 1}=\sqrt{6} \xi_{f} \sqrt{\frac{2 \Delta}{\pi h} \ln \frac{h}{\Delta}+\alpha+3 \alpha_{s o}} \ll \xi_{f 1} .
$$

Note that in situations when the approximation $d_{f} \gg \xi_{f 1}$ is not satisfied, the simple expressions (49), (54), (52), (57), (53), and (58) are not valid and to analyze the data it is convenient to introduce the effective decay length $\xi_{f 1}^{e f f}$

$$
\xi_{f 1}^{e f f}=\frac{1}{d_{m 1}-d_{m 2}} \ln \frac{I_{c m 1}}{I_{c m 2}},
$$

where $d_{m 1,2}$ are the thicknesses at which the first $\left(I_{c m 2}\right)$ and second $\left(I_{c m 2}\right)$ maxima of $I_{c}\left(d_{f}\right)$ occur.

Below we will focus on the influence of $\gamma_{B}, \tau_{s o}^{-1}$, and $\tau_{m}^{-1}$ on the critical current.

\section{B. Influence of interface transparency parameter $\gamma_{B}$ on $I_{c}$}

Consider first the simplest case of vanishing $\tau_{s o}^{-1}, \tau_{m}^{-1}$. In this limit, we immediately deduce from Eqs. (39)-(41) that

$$
\begin{aligned}
& \frac{e \xi_{f} I_{c}}{2 \pi T_{c} \sigma_{n}} \\
& \quad=\frac{T}{T_{c}} \sum_{n=0}^{\infty} \frac{\Delta^{2} G_{S}^{2}}{\omega^{2}} \\
& \quad \times \operatorname{Re}\left(\frac{a+i b}{\left[\left(\gamma_{B} \xi_{n} k\right)^{2}+G_{S}^{2}\right] \sinh \left(k d_{f}\right)+2 G_{S} \gamma_{B} \xi_{n} k \cosh \left(k d_{f}\right)}\right),
\end{aligned}
$$

where the coefficients $a$ and $b$ become

$$
a=\sqrt{|\widetilde{\omega}|+\sqrt{|\widetilde{\omega}|^{2}+1}}
$$

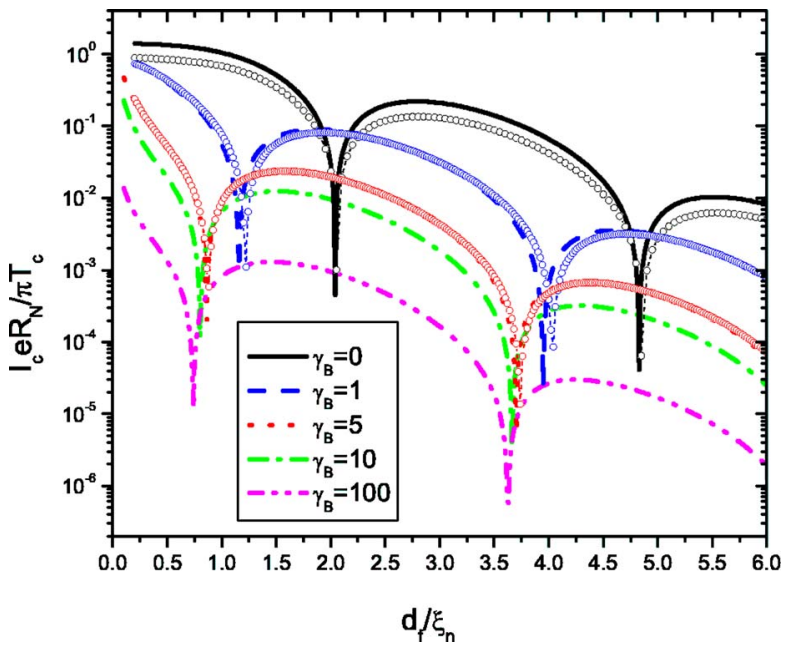

FIG. 5. (Color online) Influence of the interface transparency parameter $\gamma_{B}$ on the thickness dependence of the critical current in a S/F/S junction for $\alpha=\alpha_{s o}=0, h=3 \pi T_{c}$, and $T / T_{c}=0.5$. Open circles: the results of exact numerical calculations for $\gamma_{B}=0,1$, and 5 (from top to bottom).

$$
b=\sqrt{|\widetilde{\omega}|+\sqrt{|\widetilde{\omega}|^{2}+1}} .
$$

The dependence of the critical current as a function of the F-layer thickness calculated from Eq. (64) for $T=0.5 T_{c}$ and $h=3 \pi T_{c}$ for different values of the interface transparency parameter $\gamma_{B}$ is presented in Fig. 5. Note that for small $\gamma_{B}$ Eq. (64) is formally not applicable, since it was derived under the condition (42) of sufficiently large $\gamma_{B}$. Therefore, for $\gamma_{B}=0,1$, and 5 we have added for comparison the corresponding curves calculated numerically by direct solution of the Usadel equations for arbitrary $\gamma_{B}$, which show that Eq. (64) provides a reasonable approximation even in the small$\gamma_{B}$ range. It is clearly seen from Fig. 5 that with an increase of $\gamma_{B}$, the position of the first zero $d_{f 1}$ is shifted into the region $d_{f}<\xi_{f 1}$. As discussed above, the increase of $\gamma_{B}$ results in the suppression of $F_{ \pm f}\left( \pm d_{f}\right)$ in the $\mathrm{F}$ layer, such that $\operatorname{Re} F_{ \pm f}(x)$ changes sign in the F-layer center at smaller $d_{f}$.

The results plotted in Fig. 5 make it possible to estimate the upper limit for the $I_{c} R_{N}$ product of the SFS junction in a $\pi$ state. This upper limit can be achieved in the case of highly transparent interfaces $\left(\gamma_{B}=0\right)$ and in the absence of spin-flip and spin-orbit scattering. As follows from Fig. 5, $I_{c} R_{N}^{\max } \approx 0.1 \pi T_{c}$ that provides $I_{c} R_{N}^{\max } \approx 250 \mu \mathrm{V}$ in the case of $\mathrm{Nb}$ electrodes $\left(T_{c}=9 \mathrm{~K}\right)$.

At large F-layer thickness, $I_{c}\left(d_{f}\right)$ is determined by Eqs. (49) and (54). If spin-flip and spin-orbit scattering are negligible, the ratio $\xi_{f 1} / \xi_{f 2}=h /\left[T+\sqrt{(\pi T)^{2}+h^{2}}\right]$ follows from Eqs. (57) and (58) and depends only on $h$ and $T$. For typical ferromagnets $h \gtrsim \pi T_{c}$ and $\xi_{f 1}$ approximately equals $\xi_{f 2}$. However, if the spin-flip scattering and spin-orbit rates become relatively large $\tau_{m}^{-1}, \tau_{s o}^{-1} \gtrsim h$, the situation may change drastically. Consider first the influence of spin-flip scattering on $I_{c}$.

\section{Influence of spin-flip scattering on $I_{c}$}

If spin-flip scattering is not negligible, then the ratio of the characteristic lengths in the decaying solution (49), (54) becomes 


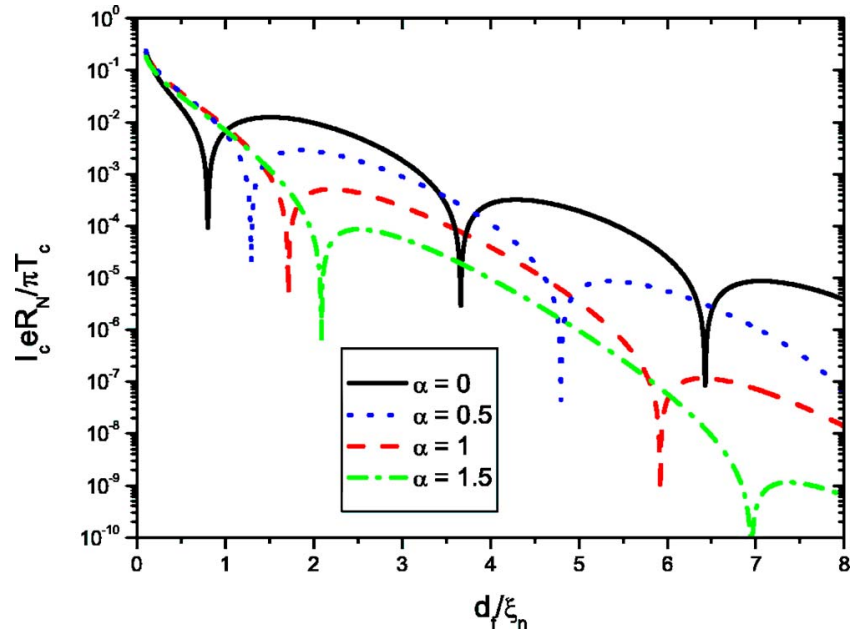

FIG. 6. (Color online) Influence of the spin-flip scattering parameter $\alpha$ on the thickness dependence of the critical current in a S/F/S junction for $\alpha_{s o}=0, h=3 \pi T_{c}, \gamma_{B}=10$, and $T / T_{c}=0.5$.

$$
\frac{\xi_{f 1}}{\xi_{f 2}}=\frac{1}{\pi T / h+\alpha+\sqrt{(\pi T / h+\alpha)^{2}+1}},
$$

and for strong spin-flip scattering $\tau_{m}^{-1} \gtrsim h$ the decay length $\xi_{f 1}$ may become much smaller than the oscillation period $\xi_{f 2}$. This results in the much stronger decrease of $I_{c}$ versus $d_{f}$ in $\mathrm{S} / \mathrm{F} / \mathrm{S}$ junctions.

The evolution of $I_{c}$ for different values of $\tau_{m}^{-1}$ calculated from Eq. (39) for $T=0.5 T_{c}, h=3 \pi T_{c}$, and $\gamma_{B}=10$ is given in Fig. 6.

One can see that with increasing $\alpha$, the critical thickness $d_{f 1}$ of the first $0-\pi$ crossover shifts to larger values of $d_{f}$.

\section{Influence of spin-orbit scattering on $\boldsymbol{I}_{\boldsymbol{c}}$}

If spin-orbit scattering is not negligible, then the ratio of the characteristic lengths in the decaying solution (49), (54) becomes

$$
\frac{\xi_{f 1}}{\xi_{f 2}}=\frac{\sqrt{1-\alpha_{s o}^{2}}}{\left(|\widetilde{\omega}|+\alpha_{s o}+\sqrt{\left.\widetilde{\omega}^{2}+2|\widetilde{\omega}| \alpha_{s o}+1\right)}\right.} .
$$

Equation (68) shows that the difference between the decaying $\xi_{f 1}$ and the oscillating length $\xi_{f 2}$ increases with $\tau_{s o}^{-1}$ even faster than for the case of spin-flip scattering. Moreover, the transition to a monotonically decaying solution takes place at $\tau_{s o}^{-1} \rightarrow h$ and $\xi_{f 2} \rightarrow \infty$.

The dependence of the critical current on $d_{f}$ calculated from Eq. (39) for $T=0.5 T_{c}, h=3 \pi T_{c}$, and $\gamma_{B}=10$ is shown in Fig. 7. One can see that the oscillation period increases strongly with increasing $\alpha_{s o}$ and diverges when $\alpha_{s o}=1$. At the same time, $d_{f 1}$ shifts to larger values of $d_{f}$.

\section{CRITICAL CURRENT OF LONG JUNCTIONS WITH TRANSPARENT INTERFACES AT ARBITRARY TEMPERATURES}

Let us now consider a S/F bilayer with a transparent interface. The complete nonlinear Usadel equation in the $\mathrm{F}$

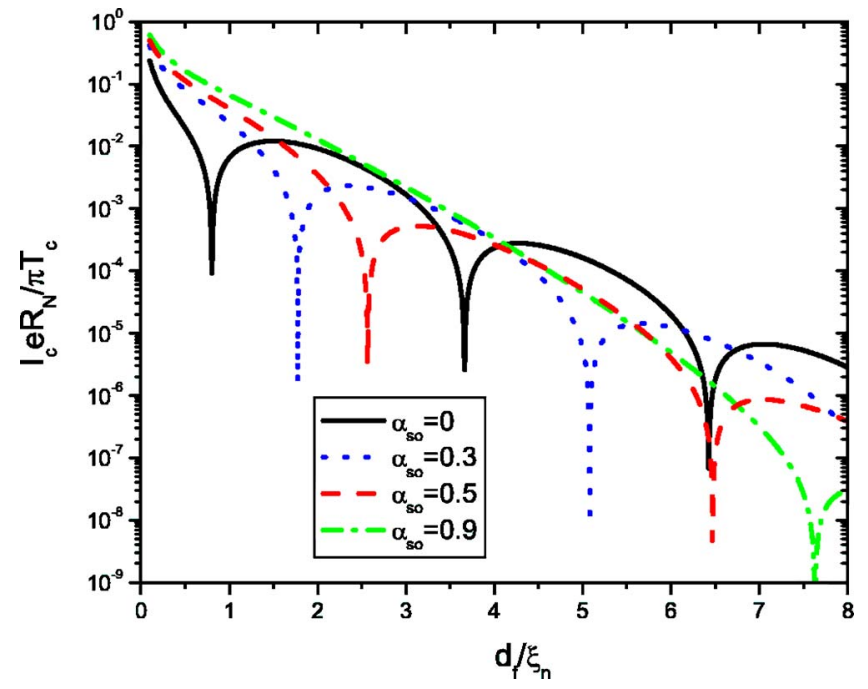

FIG. 7. (Color online) Influence of the spin-orbit scattering on the thickness dependence of the critical current in a $\mathrm{S} / \mathrm{F} / \mathrm{S}$ junction for $\alpha=0, h=3 \pi T_{c}, \gamma_{B}=10$, and $T / T_{c}=0.5$.

layer has to be employed. For the easy axis of the ferromagnet and using the usual parametrization of the normal and anomalous functions $G_{f}=\cos \theta_{f}$ and $F_{f}=\sin \theta_{f}$, it may be written in the absence of spin-orbit scattering as

$$
\xi_{n}^{2} \frac{\partial^{2}}{\partial x^{2}} \theta_{f}-\left(\frac{|\omega|+i h \operatorname{sgn}(\omega)}{\pi T_{c}}+\frac{\cos \theta_{f}}{\pi T_{c} \tau_{m}}\right) \sin \theta_{f}=0 .
$$

Note that Eq. (69) transforms into (2) in the limit of small $\theta_{f} \ll 1$.

For $\mathrm{S} / \mathrm{F} / \mathrm{S}$ junctions, this equation may be used and an analytical solution found if $d_{f} \gg \xi_{f 1}$. In that case, the decay of the Cooper pair wave function in first approximation occurs independently near each interface. ${ }^{1}$ It can therefore be treated separately enough to consider the behavior of the anomalous Green function near each $\mathrm{S} / \mathrm{F}$ interface, assuming that the F-layer thickness is infinite.

For one interface $\left(x=-d_{f} / 2\right)$, a first integral of Eq. (69) leads to

$$
\begin{aligned}
\xi_{n} \frac{d}{d x} \frac{\theta_{f}}{2}= & \sqrt{\frac{|\omega|+i h \operatorname{sgn}(\omega)}{\pi T_{c}}} \sin \frac{\theta_{f}}{2} \\
& \times \sqrt{\left(1+\frac{1}{\tau_{m}[|\omega|+i h \operatorname{sgn}(\omega)]} \cos ^{2} \frac{\theta_{f}}{2}\right)},
\end{aligned}
$$

where the boundary condition $\theta_{f}(x \rightarrow \infty)=0$ has been used. Further integration in Eq. (70) gives ${ }^{36}$

$$
\frac{\sqrt{\left[1-\varepsilon^{2} \sin ^{2}\left(\theta_{f} / 2\right)\right]}-\cos \left(\theta_{f} / 2\right)}{\sqrt{\left[1-\varepsilon^{2} \sin ^{2}\left(\theta_{f} / 2\right)\right]}+\cos \left(\theta_{f} / 2\right)}=g_{0} \exp \left(-2 q \frac{\left(d_{f} / 2+x\right)}{\xi_{f}}\right)
$$

where $k=\sqrt{2} / \xi_{f} \sqrt{[|\widetilde{\omega}|+i \operatorname{sgn}(\omega)+\alpha]}=q / \xi_{f}$, and $\varepsilon^{2}=\alpha /[|\widetilde{\omega}|$ $+i \operatorname{sgn}(\omega)+\alpha]$.

The integration constant $g_{0}$ in Eq. (71) should be determined from the boundary conditions at $\mathrm{S} / \mathrm{F}$ interfaces. For simplicity we will assume that the rigid boundary conditions are valid at $x=-d_{f} / 2$; then 


$$
\theta_{f}\left(-d_{f} / 2\right)=\arctan \frac{|\Delta|}{\omega}
$$

and from Eqs. (71) and (72). we finally get

$$
g_{0}=\frac{\left(1-\varepsilon^{2}\right) \mathcal{F}(n)}{\left[\sqrt{\left(1-\varepsilon^{2}\right) \mathcal{F}(n)+1}+1\right]^{2}}
$$

and

$$
\mathcal{F}(n)=\frac{[\Delta /(2 \pi T)]^{2}}{\left\{n+1 / 2+\sqrt{(n+1 / 2)^{2}+[\Delta /(2 \pi T)]^{2}}\right\}^{2}} .
$$

The anomalous Green function at the center of the F layer in the $\mathrm{S} / \mathrm{F} / \mathrm{S}$ junction may be taken as the superposition of the two decaying functions, ${ }^{33}$ taking into account the phase difference in each superconducting electrode,

$$
\begin{aligned}
\theta_{f}= & \frac{4}{\sqrt{1-\varepsilon^{2}}} \sqrt{g_{0}}\left[\exp \left(-q \frac{\left(d_{f} / 2+x\right)}{\xi_{f}}-i \frac{\varphi}{2}\right)\right. \\
& \left.+\exp \left(q \frac{\left(x-d_{f} / 2\right)}{\xi_{f}}+i \frac{\varphi}{2}\right)\right] .
\end{aligned}
$$

As a result, the current-phase relation is sinusoidal and the critical current becomes (see Refs. 1 and 6)

$$
\begin{aligned}
I_{c} R_{N}= & 64 \pi \frac{d_{f} T}{\xi_{f} e} \operatorname{Re}\left[\sum_{n=0}^{\infty} \frac{\mathcal{F}(n) q}{\left[\sqrt{\left(1-\epsilon^{2}\right) \mathcal{F}(n)+1}+1\right]^{2}}\right. \\
& \left.\times \exp \left(-q \frac{d_{f}}{\xi_{f}}\right)\right] .
\end{aligned}
$$

The critical current is proportional to the small factor $\exp \left(-q d_{f} / \xi_{f}\right)$. The terms neglected in our approach are much smaller and are of the order of $\exp \left(-2 q d_{f} / \xi_{f}\right)$. Therefore, they give a tiny second-harmonic term in the current-phase relation.

It should be underlined that this expression coincides with the one previously obtained in Ref. 29 in the limit $\tau_{m}^{-1} \rightarrow 0$ (no magnetic scattering) and for $T_{c} \ll h$.

In addition, note that in the limit of vanishing magnetic scattering, the temperature dependence of the critical thickness $d_{f k}$ of the $\mathrm{F}$ layer when $I_{c}=0$ may appear only through the variation of $k$ with $\omega\left(\right.$ where $k \sim \sqrt{2[|\widetilde{\omega}|+i \operatorname{sgn}(\omega)] / \xi_{f}}$ in this case). As the characteristic range of the variation of the Matsubara frequencies in the sum in (74) is $\omega \sim \pi T_{c}$, then in the limit $h \gg \pi T_{c}, \tau_{m}^{-1}$, the dependence of $d_{f k}$ on $T$ will be weak. However, when the spin scattering is not weak, i.e., $\tau_{m}^{-1} \gtrsim h$, another mechanism of the temperature dependence of $d_{f k}$ emerges due to the temperature-dependent term $\tau_{m}^{-1} \cos \theta_{f}$ in the Usadel equation (69), or, in other words, due to the complex $\omega$-dependent function $1-\varepsilon^{2}$. For a strong ferromagnet, $h \gg \pi T_{c}$, the latter mechanism may become stronger than the "thermal" one related to $k(\omega)$. It is not difficult to take into account both mechanisms in the numerical calculation of the sum (74).

As an illustration, we present in Fig. 8 the theoretical fit of the experimental data for $\mathrm{NbCu}_{0.52} \mathrm{Ni}_{0.48} \mathrm{Nb}$ junctions by Sellier et al.,${ }^{34}$ making use of Eq. (74) valid in the limit of

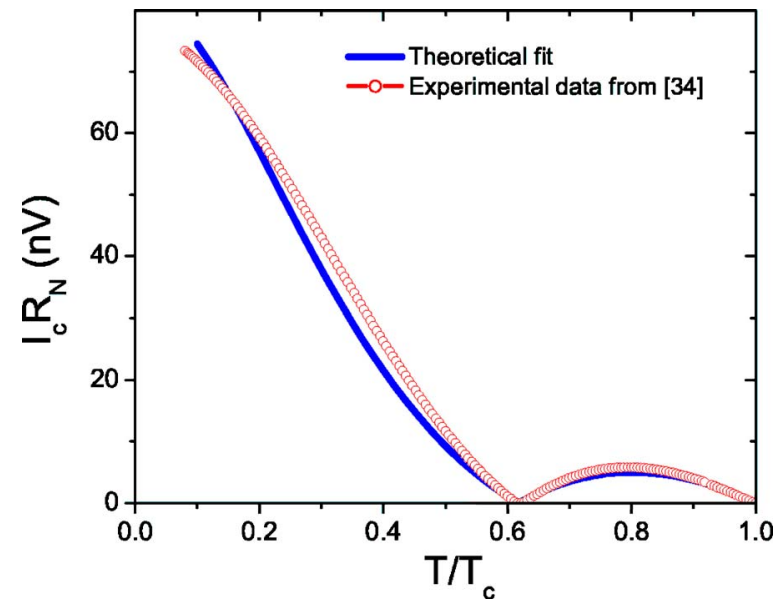

FIG. 8. (Color online) Fit to the experimental data from Ref. 34 for the critical current in a $\mathrm{NbCu}_{0.52} \mathrm{Ni}_{0.48} \mathrm{Nb}$ junction. The fitting parameters are $h=220 \mathrm{~K}$ and $\alpha=3$.

small interface resistances $\left(\gamma_{B}=0\right)$. The F-layer thickness used for the fit is $d_{f}=18 \mathrm{~nm}$ while the experimental value presented in Ref. 34 is $19 \mathrm{~nm}$. The difference may be explained by the uncertainty in the F-layer determination which is around $1 \mathrm{~nm}$, and may even increase due to the presence of a magnetically dead layer near the S/F interface. Keeping this in mind, the theoretical description of the critical current temperature dependence can be considered as rather satisfactory. In addition, Houzet et al. ${ }^{9}$ have performed numerical calculations and got also a good fit of another experimental curve of Ref. $34\left(d_{f}=17 \mathrm{~nm}\right)$ for a set of parameters which are in the same range that we have used.

As another application of the formalism, we present in Fig. 9 the theoretical fit of the experimental data of Ryazanov et al. ${ }^{35}$ for $\mathrm{NbCu}_{0.47} \mathrm{Ni}_{0.53} \mathrm{Nb}$ junctions with $d_{f}=22 \mathrm{~nm}$. Good agreement is achieved assuming $h=650 \mathrm{~K}, \alpha=1.35$, and $d_{f}$ $=21 \mathrm{~nm}$. As in the previous fit, the small difference in $d_{f}$ may be explained by the uncertainty in the F-layer determination.

Note that the rather complicated expression for the critical current (74) simplifies near $T_{c}$ and may be written as (for $\left.T_{c} \ll h\right)$

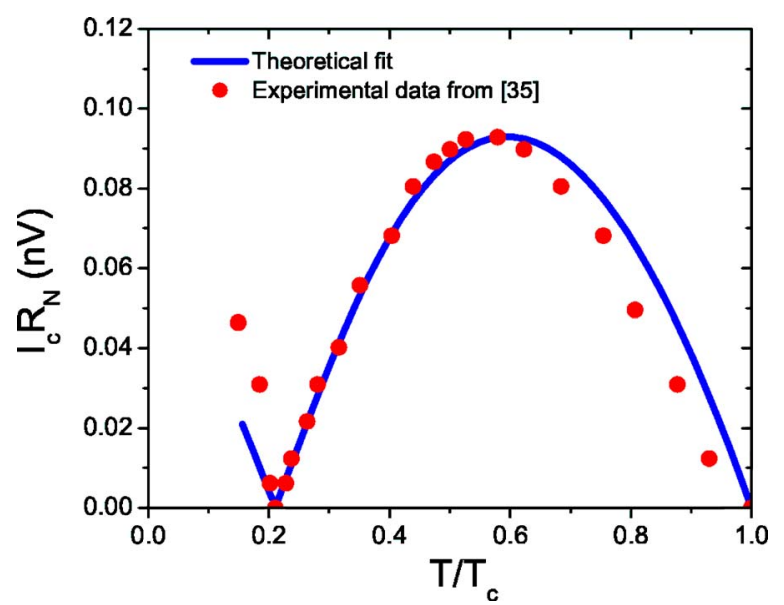

FIG. 9. (Color online) Fit to the experimental data from Ref. 35 for the critical current in a $\mathrm{NbCu}_{0.47} \mathrm{Ni}_{0.53} \mathrm{Nb}$ junction. The fitting parameters are: $h=650 \mathrm{~K}$ and $\alpha=1.35$. 


$$
I_{c} R_{N}=\frac{\Delta^{2} \pi d_{f}}{2 e T_{c} \xi_{f 2}} \frac{1}{\cos (\Psi)} \exp \left(-\frac{d_{f}}{\xi_{f 1}}\right) \sin \left(\Psi+\frac{d_{f}}{\xi_{f 2}}\right),
$$

where $\Psi$ is defined by $\tan (\Psi)=\xi_{f 2} / \xi_{f 1}$. The damping oscillatory behavior of the critical current is therefore retrieved, and the simplicity of the previous expression makes it useful for theoretical description of the evolution of the critical current with the thickness of the F layer.

\section{CONCLUSION}

We have made a detailed theoretical investigation of the influence of spin-dependent scattering in the ferromagnet on the critical temperature of $\mathrm{S} / \mathrm{F}$ multilayered systems and the critical current of S/F/S Josephson junctions. More precisely, we have demonstrated that spin-flip and spin-orbit scatterings both lead to the decrease of the decay length and the increase of the oscillation period. In addition, spin-orbit scattering may be more harmful for superconductivity than spinflip scattering. Indeed, the oscillations of $T_{c}$ and $I_{c}$ can be destroyed by spin-orbit scattering, while spin-flip scattering can only modify them. This allows us to distinguish these pair-breaking mechanisms, which both should be taken into account for theoretical fits of experimental data. Moreover, the simultaneous introduction of the spin-flip and the spinorbit scattering leads to the important prediction that the perpendicular spin-flip scattering can compensate spin-orbit scattering, and vice versa. Then the perpendicular magnetic scattering may restore the oscillations of $T_{c}$ and $I_{c}$, which would otherwise be absent if spin-orbit scattering is strong enough.

We have also studied the influence of the interface transparency on $T_{c}$ and $I_{c}$ in $\mathrm{S} / \mathrm{F}$ and $\mathrm{S} / \mathrm{F} / \mathrm{S}$ structures, respectively. The nonmonotonic behavior of $T_{c}$ with the interface transparency parameter $\gamma_{B}$ was predicted in the case of thin $\mathrm{F}$ layers due to multiple scattering at the interfaces. We have shown that the same mechanism is responsible for the $0-\pi$ transition in $\mathrm{S} / \mathrm{F} / \mathrm{S}$ junctions with small thickness $d_{f}$ in the case of large $\gamma_{B}$. For larger $d_{f}$ a detailed analytical and numerical study of the influence of $\gamma_{B}$ parameter is presented.

It was predicted in Ref. 3 that in the case of noncollinear magnetic ordering the long-ranged triplet component of the superconducting condensate could be generated. The spinorbit and the perpendicular magnetic scattering are rather harmful for this long-ranged triplet component. Our analysis shows that ferromagnetic alloys like $\mathrm{Cu}_{x} \mathrm{Ni}_{1-x}$, where following our estimates the parameter $1 / h \tau_{m}$ may exceed unity, are not suitable candidates for the experimental search for the triplet component.

Finally, note that it may be interesting to study Josephson junctions with the ferromagnetic layer substituted by a paramagnetic one. Applying an external magnetic field $H$, it is possible to produce the polarization of the magnetic atoms and then generate an internal field $h \sim \chi H$, where $\chi$ is the paramagnetic susceptibility. The variation of the external field allows one to change the relative contribution of the scattering mechanisms and the exchange field and then to modify the properties of the junction in a controllable way. In particular, it could provoke the transition from the 0 to $\pi$ state.

\section{ACKNOWLEDGMENTS}

The authors thank M. Daumens, M. Houzet, M. Kulic, C. Meyers, and V. V. Ryazanov for useful discussions. We also thank H. Sellier and V. V. Ryazanov for communication of their experimental data. This work was supported in part by EGIDE Program 10197RC, ESF PI-Shift Program, RFBR Grant No. 06-02-90865, NanoNed program under Project No. TCS.7029, and NWO-RFBR program under Project No. 047.016.005.

\section{APPENDIX}

The coefficients $A_{\omega \pm}$ and $B_{\omega \pm}$ that appear in Eqs. (33) and (34) may be written as

$$
\begin{aligned}
& A_{\omega+}=\frac{2 \Delta G_{S} \cos (\varphi / 2)}{|\omega|\left[\gamma_{B} \xi_{n} k_{+} \sinh \left(k_{+} d_{f}\right)+\left|G_{S}\right| \cosh \left(k_{+} d_{f}\right)\right]\left(1+\eta_{\omega}^{2}\right)}, \\
& B_{\omega+}=\frac{2 i \Delta G_{S} \sin (\varphi / 2)}{|\omega|\left[\gamma_{B} \xi_{n} k_{+} \cosh \left(k_{+} d_{f}\right)+\left|G_{S}\right| \sinh \left(k_{+} d_{f}\right)\right]\left(1+\eta_{\omega}^{2}\right)}, \\
& A_{\omega-}=-\frac{2 \Delta G_{S} \eta_{\omega} \cos (\varphi / 2)}{|\omega|\left[\gamma_{B} \xi_{n} k_{-} \sinh \left(k_{-} d_{f}\right)+\left|G_{S}\right| \cosh \left(k_{-} d_{f}\right)\right]\left(1+\eta_{\omega}^{2}\right)}, \\
& B_{\omega-}=-\frac{2 i \Delta G_{S} \eta_{\omega} \sin (\varphi / 2)}{|\omega|\left[\gamma_{B} \xi_{n} k_{-} \cosh \left(k_{-} d_{f}\right)+\left|G_{S}\right| \sinh \left(k_{-} d_{f}\right)\right]\left(1+\eta_{\omega}^{2}\right)} .
\end{aligned}
$$

The symmetry relations following from Eqs. (33)-(35) are at $h \leqslant \tau_{s o}^{-1}$

$$
\begin{gathered}
\eta_{\omega}=\eta_{-\omega}^{*}, \quad A_{-\omega+}^{*}=A_{\omega+}, \quad A_{-\omega-}^{*}=A_{\omega-}, \\
B_{-\omega+}^{*}=-B_{\omega+}, \quad B_{-\omega-}^{*}=-B_{\omega-},
\end{gathered}
$$

and if $h \geqslant \tau_{s o}^{-1}$

$$
\begin{aligned}
\eta_{-\omega}^{*} \eta_{\omega} & =-1, \quad \beta_{\omega+}=\beta_{\omega-}^{*}=\beta, \quad \eta_{-\omega}^{*} A_{-\omega+}^{*}=A_{\omega-}, \quad A_{-\omega-}^{*} \\
& =\eta_{\omega} A_{\omega+}, \quad \eta_{-\omega}^{*} B_{-\omega+}^{*}=-B_{\omega-}, \quad B_{-\omega-}^{*}=-\eta_{\omega} B_{\omega+} .
\end{aligned}
$$

These relations allow us to simplify the calculations for the determination of the critical current. 
${ }^{1}$ A. I. Buzdin, Rev. Mod. Phys. 77, 935 (2005).

${ }^{2}$ A. A. Golubov, M. Yu. Kupriyanov, and E. Il'ichev, Rev. Mod. Phys. 76, 411 (2004).

${ }^{3}$ F. S. Bergeret, A. F. Volkov, and K. B. Efetov, Rev. Mod. Phys. 77, 1321 (2005).

${ }^{4}$ V. V. Ryazanov, V. A. Oboznov, A. Yu. Rusanov, A. V. Veretennikov, A. A. Golubov, and J. Aarts, Phys. Rev. Lett. 86, 2427 (2001).

${ }^{5}$ H. Sellier, C. Baraduc, F. Lefloch, and R. Calemczuk, Phys. Rev. B 68, 054531 (2003).

${ }^{6}$ V. A. Oboznov, V. V. Bol'ginov, A. K. Feofanov, V. V. Ryazanov, and A. I. Buzdin, cond-mat/0508573 (unpublished).

${ }^{7}$ G. Eilenberger, Z. Phys. 214, 195 (1968).

${ }^{8}$ L. Usadel, Phys. Rev. Lett. 25, 507 (1970).

${ }^{9}$ M. Houzet, V. Vinokur, and F. Pistolesi, Phys. Rev. B 72, 220506(R) (2005).

${ }^{10}$ P. Fulde and K. Maki, Phys. Rev. 141, 275 (1966).

${ }^{11}$ A. A. Abrikosov, L. P. Gor'kov, and I. E. Dzyaloshinski, Methods of Quantum Field Theory in Statistical Physics (Dover, New York, 1975).

${ }^{12}$ A. I. Buzdin, Pis'ma Zh. Eksp. Teor. Fiz. 42, 283 (1985) [JETP Lett. 42, 350 (1985)].

${ }^{13}$ A. I. Buzdin and M. Y. Kuprianov, Pis'ma Zh. Eksp. Teor. Fiz. 52, 1089 (1990) [JETP Lett. 52, 487 (1990)].

${ }^{14}$ Z. Radovic, M. Ledvij, L. Dobrosavljevic-Grujic, A. I. Buzdin, and J. R. Clem, Phys. Rev. B 44, 759 (1991).

${ }^{15}$ E. A. Demler, G. B. Arnold, and M. R. Beasley, Phys. Rev. B 55, 15174 (1997).

${ }^{16}$ S. Oh, Y. Kim, D. Youm, and M. R. Beasley, Phys. Rev. B 63, 052501 (2000).

${ }^{17}$ L. R. Tagirov, Physica C 307, 145 (1998).

${ }^{18}$ V. I. Fal'ko, A. F. Volkov, and C. Lambert, Phys. Rev. B 60, 15394 (2002).
${ }^{19}$ A. Millis, D. Rainer, and J. A. Sauls, Phys. Rev. B 38, 4504 (1988).

${ }^{20}$ M. Fogelström, Phys. Rev. B 62, 11812 (2000).

${ }^{21}$ J. C. Cuevas and M. Fogelström, Phys. Rev. B 64, 104502 (2001).

${ }^{22}$ M. Eschrig, J. Kopu, J. C. Cuevas, and Gerd Schön, Phys. Rev. Lett. 90, 137003 (2003).

${ }^{23}$ A. I. Buzdin, A. V. Vedyayev, and N. V. Ryshanova, Europhys. Lett. 48, 686 (1999).

${ }^{24}$ I. Baladié and A. Buzdin, Phys. Rev. B 67, 014523 (2003).

${ }^{25}$ M. Yu. Kuprianov and V. F. Lukichev, Zh. Eksp. Teor. Fiz. 94, 139 (1988) [Sov. Phys. JETP 67, 1163 (1988)].

${ }^{26}$ J. Aarts, J. M. E. Geers, E. Brüeck, A. A. Golubov, and R. Coehoorn, Phys. Rev. B 56, 2779 (1997).

${ }^{27}$ Ya. V. Fominov, N. M. Chtchelkatchev, and A. A. Golubov, Phys. Rev. B 66, 014507 (2002).

${ }^{28}$ A. I. Buzdin, L. N. Bulaevskii, and S. V. Panyukov, Pis'ma Zh. Eksp. Teor. Fiz. 35, 147 (1982) [JETP Lett. 35, 178 (1982)].

${ }^{29}$ A. I. Buzdin and M. Y. Kuprianov, Pis'ma Zh. Eksp. Teor. Fiz. 53, 308 (1991) [JETP Lett. 53, 321 (1991)].

${ }^{30}$ V. N. Krivoruchko and R. V. Petryuk, Phys. Rev. B 66, 134520 (2002).

${ }^{31}$ F. S. Bergeret, A. F. Volkov, and K. B. Efetov, Phys. Rev. B 68, 064513 (2003).

${ }^{32}$ A. I. Buzdin, Pis'ma Zh. Eksp. Teor. Fiz. 53, 308 (1991). [JETP Lett. 78, 583 (2003)].

${ }^{33}$ K. K. Likharev, Rev. Mod. Phys. 51, 101 (1979).

${ }^{34}$ H. Sellier, C. Baraduc, F. Lefloch, and R. Calemczuk, Phys. Rev. Lett. 92, 257005 (2004).

${ }^{35}$ V. V. Ryazanov, V. A. Oboznov, A. S. Prokofiev, V. V. Bolginov, and A. K. Feofanov, J. Low Temp. Phys. 136, 385 (2004).

${ }^{36}$ L. Cretinon, A. K. Gupta, H. Sellier, F. Lefloch, M. Faurý, A. Buzdin, and H. Courtois, Phys. Rev. B 72, 024511 (2005). 